\title{
Adaptação cultural e validação dos construtos: serviço logístico, satisfação e lealdade
}

\section{Cultural adaptation and validation analysis of the constructs: logistics service, satisfaction, and loyalty}

\author{
Alexandre Fun Ghi Su${ }^{1}$ \\ Mauro Sampaio ${ }^{1}$
}

\begin{abstract}
Resumo: Este estudo apresenta o processo de adaptação cultural dos construtos serviço logístico, satisfação e lealdade à língua portuguesa. A adaptação cultural inclui as fases: tradução, retrotradução, revisão por comitê, pré-teste e análise de confiabilidade e validade. Os resultados mostram que há evidências de que as medidas utilizadas são confiáveis, porém foi necessário excluir 11 itens da relação de 35 itens teóricos identificados na literatura. Também há evidências de que as medidas são válidas como instrumentos de pesquisa em português pelos métodos de: análise de conteúdo; validade convergente; validade discriminante; análise fatorial exploratória; e análise fatorial confirmatória.
\end{abstract}

Palavras-chave: Confiabilidade. Estatística multivariada. Metodologia de pesquisa.

\begin{abstract}
This study aims to present the cross-cultural adaptation process of the constructs logistic service, satisfaction, and loyalty to the Brazilian culture. This adaptation process included the steps of translation, back translation, review by expert committee, pretesting, and analysis of validity and reliability. The study suggests that the constructs logistics service, satisfaction, and loyalty are reliable according to the Brazilian culture, but it was necessary to exclude 11 items from the list of 35 items identified in the theory. There is also evidence that the measures are valid as research instruments in Portuguese by the following methods: content analysis, convergent validity, discriminant validity, exploratory factor analysis, and confirmatory factor analysis.
\end{abstract}

Keywords: Reliability. Multivariate statistics. Methods of research.

\section{Introdução}

A disponibilidade de medidas confiáveis e válidas, segundo padrões científicos, é um importante passo para o desenvolvimento de uma área do conhecimento (VARGAS et al., 2008; PEREIRA; PINTO, 2011; ALVES; BORNIA, 2011). Entretanto, muitos dos instrumentos de medida desenvolvidos em logística foram criados em países norte-americanos e europeus, carecendo de comprovação de confiabilidade e validade no ambiente da língua portuguesa.

Os pesquisadores brasileiros interessados em pesquisas quantitativas percorrem diferentes caminhos: ou desenvolvem uma escala completamente nova, correndo o risco de ignorar avanços da área de conhecimento; ou utilizam uma escala traduzida, sem maiores preocupações com questões de confiabilidade e validade. Ambos os caminhos não são recomendados. $O$ processo correto de adaptação cultural envolve etapas mais elaboradas que somente uma tradução. Craig e Douglas (2006) afirmam que a adaptação cultural de medidas é fundamental para evitar um viés na interpretação dos resultados.

O objetivo deste trabalho é entender, descrever e avaliar o processo de adaptação cultural dos construtos serviço logístico (MENTZER; FLINT; HULT, 2001), satisfação (SELNES; GONHAUG, 2000) e lealdade (STANK et al., 2003; CARUANA, 2002) para a língua portuguesa, bem como análise de sua validade e confiabilidade. Existem diversas outras medidas passíveis de investigação, porém este trabalho focou nestes construtos específicos pelo interesse dos autores em aplicar estas medidas em estudos posteriores.

Em síntese, pretende-se responder às seguintes questões de pesquisa: Até que ponto os construtos serviço logístico, satisfação e lealdade são confiáveis como instrumento de pesquisa? Até que ponto os construtos serviço logístico, satisfação e lealdade são válidos como instrumento de pesquisa?

\footnotetext{
${ }^{1}$ Departamento de Engenharia de Produção, Centro Universitário da FEI, CEP 09850-901, São Bernardo do Campo, SP, Brasil, e-mail: alexandre@uol.com.br; msampaio@ fei.edu.br
} 
Para tanto, o questionário em português, contendo os construtos de interesse, foi aplicado em 152 profissionais de compras das principais empresas químicas do Brasil. Foram avaliados o nível de serviço logístico, a satisfação e a lealdade dos principais fornecedores de itens de alavancagem, que são aqueles itens que impactam de forma significativa os custos e podem se obtidos de vários fornecedores (KRALJIC, 1983).

Este estudo está dividido em cinco seções, incluindo esta introdução. A segunda seção realiza uma revisão da literatura dos construtos serviço logístico, satisfação e lealdade. A terceira seção apresenta o processo de adaptação cultural e análise de confiabilidade e validade. A quarta seção mostra os resultados obtidos. A última seção apresenta as conclusões do estudo, as limitações e sugestões para trabalhos futuros.

\section{Revisão da literatura}

Esta seção apresenta uma revisão da evolução da literatura sobre os construtos: serviço logístico, satisfação, lealdade.

\subsection{Serviços logísticos}

Serviço Logístico é uma medida de quão bem um sistema se comporta ao fornecer um produto ao cliente em tempo, espaço e forma (STERLING; LAMBERT, 1987). Até os meados dos anos 1990, o serviço logístico era mensurado por medidas objetivas, operacionais e internas (LAMBERT; COOPER; PAGH, 1998; MENTZER; FLINT; HULT, 2001) como: nível de disponibilidade, tempo de ciclo do pedido, pontualidade, entre outras. A percepção do cliente em relação ao desempenho do serviço logístico do fornecedor não era avaliada (MENTZER; FLINT; HULT, 2001). A partir dos anos 2000, as pesquisas passaram a investigar, além dos aspectos operacionais, também a percepção dos clientes. Trajetória esta similar à percorrida por Grönroos (1984) e Parasuraman, Zeithaml e Berry $(1985,1988)$; Salomi, Miguel e Abackerli (2005), na avaliação da qualidade de serviços.

Mentzer, Flint e Hult (2001) desenvolveram uma escala para o serviço logístico, inspirada em Stank et al. (2003) e Stank, Goldsby e Vickery (1999), baseada na percepção do cliente tanto de aspectos operacionais quanto de aspectos relacionais, conforme mostra o Quadro 1. A dimensão "Desempenho Operacional" mensura a capacidade de o fornecedor entregar seus produtos de forma pontual e confiável e inclui aspectos como: disponibilidade, prazo de entrega e agilidade na entrega, e também confiabilidade. A dimensão "Desempenho Relacional" mensura a habilidade do fornecedor de entender as necessidades e expectativas do cliente, trata-se de uma dimensão externa e orientada ao mercado (COLLIER, 1991). Diversas pesquisas anteriores não consideraram o componente relacional do serviço logístico (DAVIS, 2006). O Anexo 1 apresenta os itens traduzidos de cada construto.

Stank, Goldsby e Vickery (1999), Stank et al. (2003), Davis-Sramek, Mentzer e Stank (2008) e Davis-Sramek et al. (2009) encontraram uma relação causal unidirecional significativa entre as dimensões: desempenho relacional e desempenho operacional. Entretanto, a relação causal inversa, o impacto do desempenho operacional sobre o desempenho relacional não foi identificado.

No Brasil, o estudo de Tontini e Zanchett (2010) mostrou que o serviço logístico de uma empresa de pequeno porte era composto por cinco dimensões: confiabilidade, responsividade, flexibilidade, segurança e empatia. Tais dimensões podem ser compreendidas como variáveis observáveis das variáveis latentes: desempenho relacional e operacional definidas por Stank et al.(2003).

\subsection{Satisfação}

O construto satisfação já foi conceituado, mensurado e testado na literatura em várias indústrias e contextos (OLIVER, 1980; PARASURAMAN; ZEITHAML; BERRY, 1985; DAVIS-SRAMEK; MENTZER; STANK, 2008). Normalmente é utilizada a confirmação de expectativas como base para a definição operacional do construto satisfação.

O construto satisfação pode ser conceituado segundo dois aspectos: transacional ou acumulado. $\mathrm{O}$ aspecto transacional é a percepção do desempenho do cliente em relação à troca mais recente (OLIVER, 1993). Outros autores, como Anderson, Fornell e Lehmann (1994), afirmam que a satisfação deve ser avaliada por um julgamento da experiência acumulada referente a um relacionamento de

Quadro 1. Definição das dimensões do construto serviço logístico investigadas nesta pesquisa.

\begin{tabular}{|c|l|l|}
\hline \multicolumn{1}{|c|}{ Dimensões } & \multicolumn{1}{|c|}{ Definição } & \multicolumn{1}{|c|}{ Fontes } \\
\hline Desempenho Operacional (DO) & $\begin{array}{l}\text { Dimensão que avalia a capacidade do fornecedor em } \\
\text { entregar produtos e serviços dentro de um padrão } \\
\text { logístico pré-acordado. }\end{array}$ & $\begin{array}{l}\text { Adaptado de Stank, Goldsby } \\
\text { e Vickery (1999), Stank et al. } \\
\text { (2003) e Mentzer, Flint e }\end{array}$ \\
\hline Desempenho Relacional (DR) & $\begin{array}{l}\text { Dimensão que avalia a habilidade do fornecedor em } \\
\text { entender as necessidades e expectativas do cliente. }\end{array}$ & Hult (2001). \\
\hline
\end{tabular}


trocas. Essa concepção é mais apropriada, pois a satisfação acumulada se correlaciona positivamente com a lealdade de clientes (FORNELL, 1992; REICHHELD; SASSER, 1990). O Quadro 2 apresenta a definição de satisfação utilizada nesta pesquisa e o Anexo 1 apresenta os itens traduzidos de cada construto.

Também foi identificado que o serviço logístico é um importante antecedente e preditor do nível de satisfação do cliente (DAUGHERTY; STANK; ELLINGER, 1998; LEUTHESSER; KOHLI, 1995). No Brasil, vários autores (RIBEIRO; MACHADO; TINOCO, 2010; BRANCO; RIBEIRO; TINOCO, 2010; FLEURY; SILVA, 1997) utilizaram medidas de satisfação similares em seus respectivos estudos.

\subsection{Lealdade}

O construto lealdade mensura não apenas componente comportamental, caracterizado pela repetição da compra, mas também um componente psicológico (OLIVER, 1999).

De acordo com essa visão, não é correto fazer qualquer inferência sobre lealdade apenas com base em padrões de compra repetida, uma vez que a lealdade verdadeira inclui também uma dimensão atitudinal que avalia a disposição emocional do cliente em manter o relacionamento com seu fornecedor." (LARÁN; ESPINOZA, 2004, p. 52).

Em situações de monopólio, os clientes são forçados a comprar de um fornecedor, porém podem trocá-lo a qualquer momento em uma situação da emergência de novos players no mercado (KUMAR; BOHLING; LADDA, 2003). O construto lealdade possui, portanto, duas dimensões distintas: atitudinal e comportamental, conforme mostra o Quadro 3. O Anexo 1 apresenta os itens traduzidos de cada construto.

Kandampully (1998) reforça que o relacionamento leal e verdadeiro entre empresas e clientes é criado pela capacidade do fornecedor de estabelecer relacionamento de longo prazo e se conectar emocionalmente com seus clientes. Por essa razão, a verdadeira lealdade deve ser determinada não somente pelo comportamento de recompra, mas pelo sentimento e emoção provenientes do relacionamento (CHANDHURI; HOLBROOK, 2001). Dick e Basu (1994) e Davis-Sramek, Mentzer e Stank (2008) definem lealdade como a relação causal entre lealdade atitudinal e lealdade comportamental.

Diversos pesquisadores têm comprovado que a lealdade atitudinal tem um impacto positivo sobre lealdade comportamental (RUYTER; KO; LEMMINK, 2001; WETZELS; RUYTER; BIRGELEN, 1998; DAVIS-SRAMEK; MENTZER; STANK, 2008).

\section{Metodologia}

O trabalho foi realizado em duas fases: estudo da adaptação cultural (fase 1) e análise da confiabilidade e validade (fase 2). Esta seção descreve os seguintes pontos: procedimentos de adaptação cultural, pré-teste do instrumento de pesquisa, população, amostra, coleta de dados e apresentação das técnicas adotadas para análise de confiabilidade e validade.

\subsection{Procedimentos de adaptação cultural}

Esta fase foi conduzida de acordo com os métodos preconizados na literatura por Cha, Kim e Erlen (2007). A tradução do instrumento do inglês para o português foi realizada de forma

Quadro 2. Definição do construto satisfação investigada nesta pesquisa.

\begin{tabular}{|l|l|l|}
\hline \multicolumn{1}{|c|}{ Dimensão } & \multicolumn{1}{|c|}{ Definição } & \multicolumn{1}{c|}{ Fontes } \\
\hline Satisfação & $\begin{array}{l}|c| \\
\text { (ST) }\end{array}$ & $\begin{array}{l}\text { Adaptado de Selnes e Gonhaug } \\
\text { (2000), Garbarino e Johnson }\end{array}$ \\
& contentamento do cliente em relação ao desempenho \\
do fornecedor. & $\begin{array}{l}\text { (1999), Seiders et al. (2005), } \\
\text { Keiningham et al. (2007) e } \\
\text { Olsen (2007). }\end{array}$ \\
\hline
\end{tabular}

Quadro 3. Definição das dimensões do construto lealdade investigada nesta pesquisa.

\begin{tabular}{|l|l|l|}
\hline \multicolumn{1}{|c|}{ Dimensões } & \multicolumn{1}{|c|}{ Definições } & \multicolumn{1}{c|}{ Fontes } \\
\hline $\begin{array}{l}\text { Lealdade Atitudinal } \\
\text { (LA) }\end{array}$ & $\begin{array}{l}\text { Mensura a vontade do cliente em manter um } \\
\text { relacionamento próximo com o fornecedor no futuro. }\end{array}$ & $\begin{array}{l}\text { Adaptado de Caruana (2002), } \\
\text { Kim e Frazier (1997), Kumar, } \\
\text { Hibbard e Stern (1994) e } \\
\text { Stank et al. (2003). }\end{array}$ \\
\hline $\begin{array}{l}\text { Lealdade comportamental } \\
\text { (LC) }\end{array}$ & $\begin{array}{l}\text { Mensura a probabilidade de o cliente recomprar do } \\
\text { mesmo fornecedor no futuro. Intenção de recompra. }\end{array}$ & $\begin{array}{l}\text { Adaptado de Caruana (2002), } \\
\text { Matilla (2001) e Too, Souchon } \\
\text { e Thirkell (2001). }\end{array}$ \\
\hline
\end{tabular}


independente por dois tradutores brasileiros que conheciam os objetivos do estudo. As duas versões em português, após o consenso, geraram uma única versão, denominada Questionário 1, sendo esta, então, submetida à retrotradução, realizada por outros dois tradutores residentes nos Estados Unidos, com domínio dos idiomas português e inglês. Eles não tiveram contato com o instrumento original, gerando, assim, a versão Questionário 2. Subsequentemente, esta versão foi submetida a um comitê de juízes, composto por quatro especialistas em logística, com domínio do idioma inglês. Eles compararam cada item dos construtos originais com a versão traduzida, em relação às equivalências semântico-idiomáticas, com o intuito de garantir a tradução correta. Depois desse processo, foi gerada a versão Questionário 3, submetida a outro comitê composto por quatro professores especialistas em elaboração de escalas. Apenas a versão Questionário 3 estava disponível e eles tiveram que verificar a equivalência cultural, que está relacionada com o contexto e experiências de vida dos brasileiros, e a equivalência conceitual, que é a verificação da manutenção dos conceitos dos construtos originais na versão traduzida. Foram considerados consistentes itens com $80 \%$ de concordância em todas as análises de equivalência. Outra versão foi gerada após essas análises, Questionário 4, que foi aplicada a 10 compradores da indústria química que responderam o instrumento e sugeriram modificações em alguns itens para melhorar a compreensão. A versão Questionário 5 foi submetida às análises de confiabilidade e validade. O Anexo 1 apresenta a definição operacional final dos construtos equivalente ao Questionário 5.

\subsection{Pré-teste do instrumento de pesquisa}

Para realizar o pré-teste de campo, este trabalho utilizou um método de pesquisa tipo survey on-line, de corte transversal (PARASURAMAN; GREWAL; KRISHNAN, 2006), com amostra não probabilística da população de interesse. Os respondentes foram profissionais da área de compras de empresas químicas que compram materiais e serviços para suas respectivas empresas. Estes compradores avaliaram o relacionamento da empresa química com seu principal fornecedor de um item de alavancagem, ou seja, um item de elevado custo para a empresa e fornecido por mais de uma empresa.

\subsubsection{População e amostra}

A população selecionada foi a de compradores das empresas do setor químico nacional, todas as empresas com receita operacional bruta superior a 1,0 bilhão de dólares no ano base de 2009, segundo dados da revista "Maiores e Melhores" divulgados no site exame.com. O motivo do foco nesta indústria foi para garantir maior homogeneidade das necessidades de clientes e serviços prestados pelos fornecedores, além do interesse dos autores em realizar pesquisas futuras neste segmento. Foi realizada uma amostragem não probabilística por conveniência, uma vez que não existe um banco de dados disponível com a relação de todos esses profissionais. Hair et al. (2009) afirmam que a chance de um elemento da população ser selecionado é desconhecida com amostras não probabilísticas. Em amostras como esta, por conveniência (HAIR et al., 2009), a seleção dos elementos da amostra é feita entre os indivíduos que estão mais disponíveis para participar do estudo e que sejam competentes em fornecer as informações requeridas. Apesar de não ser uma amostra representativa da população de empresas químicas em geral, os compradores são representantes das maiores empresas químicas do Brasil e capazes de fornecer as informações desejadas nesta pesquisa.

De qualquer forma, o propósito da pesquisa foi avaliar a confiabilidade e a validade dos construtos de interesse e não a estrutura de relações. Sendo assim, a representatividade da amostra não é questão crucial. Em casos como este, uma adaptação cultural inicial, é até desejável que a amostra seja mais homogênea, a fim de evitar efeitos de moderadores imprevistos que possam influenciar nas relações estudadas (HAIR et al., 2009). É importante ressaltar que a validação de um instrumento não pode ser esgotada em um único estudo (HAIR et al., 2009). Portanto, estudos posteriores são convenientes.

\subsubsection{Coleta de dados}

A versão brasileira do questionário foi aplicada entre janeiro e março de 2011. Esses compradores avaliaram alguns de seus principais fornecedores de itens de alavancagem (KRALJIC, 1983). O questionário foi autoadministrado. Foram convidados 152 compradores para participar da pesquisa. Seguindo a sugestão de Dillman (2000), àqueles que não responderam à pesquisa foram enviados dois e-mails solicitando participação. Dos 152 e-mails convites enviados, 7 e-mails retornaram como "não entregues". Desse total, 56 compradores responderam à pesquisa. A taxa de resposta foi de $38,6 \%$, já descontando os compradores que não foram contatados. Um total de 148 questionários foi preenchido completamente. A maioria dos compradores respondeu mais de um questionário, mas cada um corresponde a uma relação cliente-fornecedor diferente. Em média, foram 2,6 questionários por comprador (a).

Em termos de tamanho da amostra, embora não existam muitas indicações empíricas ou teóricas sobre o que seja uma boa amostra, a referência adotada é a 
de Baumgartner e Homburg (1996, p. 146), que sugere que a razão entre o número de casos da amostra e o número de parâmetros livres deve ser compreendida entre "5:1 e 10:1", para obter estimativas de parâmetros confiáveis. A presente pesquisa cumpre esse critério, uma vez que estimam, ao final, 28 parâmetros com uma amostra de calibração de 148 casos, isto é, uma razão de 5:1.

\subsubsection{Análises de confiabilidade e validade}

Foram executados os seguintes procedimentos de análise de dados:

- Preparação: foram realizadas as análises de estatística descritiva, observações atípicas, verificação da normalidade e linearidade.

- Confiabilidade: representa a reprodutividade dos resultados obtidos pelos construtos em diferentes condições. A confiabilidade foi testada pela análise da consistência interna das dimensões de cada construto com a utilização do coeficiente alfa de Cronbach.

- Validade: é a propriedade do construto em medir o que se propõe. Tendo como base os dados coletados na avaliação de equivalência conceitual, a validade de conteúdo foi determinada com a utilização do índice de avaliação de conteúdo (IAC), sendo recomendado que o IAC seja superior a $80 \%$ (NOVATO; GROSSI; KIMURA, 2008).

A validade convergente examina se as variáveis observáveis de um constructo estão relacionadas entre si. Esta validade foi determinada pela correlação dos escores obtidos entre os construtos estudados. A validade convergente também foi comprovada pela análise fatorial exploratória e análise fatorial confirmatória.

A validade discriminante examina se as variáveis observáveis de um constructo estão relacionadas a outros construtos. Entre os vários métodos para estabelecer a validade discriminante, Fornell e Larcker (1981) recomendam a comparação da variância extraída de cada construto com as variâncias compartilhadas (o quadrado do coeficiente de correlação) entre os construtos. $\mathrm{O}$ valor da variância compartilhada deve ser menor que o da variância extraída na maioria dos construtos.

Foram utilizados os softwares SPSS Statistics 21 e LISREL 8.80 for Windows para realizar as análises univariadas e multivariadas dos dados obtidos.

\section{Resultados}

Nesta seção, são apresentados os resultados da adaptação cultural, a preparação dos dados (estatística descritiva, observações atípicas, normalização, linearidade) e análise das propriedades psicométricas da amostra coletada.

\subsection{Adaptação cultural}

Nesta fase, alterações nos itens dos contrutos foram introduzidas com as sugestões dos especialistas e profissionais da área. O Anexo 1 apresenta os itens traduzidos de cada construto. As respostas dos compradores foram registradas em escalas Likert com sete possibilidades de respostas, variando de (1) "pior" a (7) "melhor" ou (1) "discordo completamente" a (7) "concordo completamente", dependendo da escala.

\subsection{Preparação da análise}

Antes do início do processo de análise de confiabilidade e validade, foi realizada uma verificação prévia dos dados, com vistas a identificar erros de codificação, assegurar que as variáveis com escalas invertidas realmente fossem recodificadas e identificar eventuais observações atípicas.

No que concerne às perguntas não respondidas, foi adotada a abordagem listwise (VIEIRA, 2009), ou seja, os casos com não respostas $(1,3 \%)$ foram eliminados. Como poucos casos (dois casos) foram eliminados, esta questão não chegou a comprometer a análise posterior.

\subsubsection{Observações atípicas}

A observação atípica é uma combinação única de características identificáveis, sendo notadamente diferentes das outras observações (HAIR et al., 2009). A identificação das observações atípicas foi realizada pelos métodos de detecção univariada, por diagramas de dispersão bivariados e pelo cálculo da medida $\mathrm{D}^{2}$ de Mahalanobis. Essa medida avaliou a posição de cada observação, comparada com o centro de todas as observações, em um conjunto de variáveis. Como resultado desses testes, nenhuma observação foi eliminada. Nenhuma observação foi considerada extrema em um número suficiente de variáveis para ser considerada não representativa da população. Algumas observações foram identificadas como atípicas, mas não diferentes o suficiente para serem retiradas da amostra e consideradas não representativas da população.

\subsubsection{Normalização}

Neste estudo, todas as variáveis foram mensuradas em uma escala likert de sete pontos, que, do ponto de vista técnico, é uma escala ordinal. Porém, neste estudo, foi considerada como uma escala intervalar, prática generalizada em investigação em ciências sociais (VIEIRA, 2009; HILL; HILL, 
2000; KINNEAR; TAYLOR, 1991). As variáveis em análise são de fato contínuas, no entanto, só é possível medi-las por meio de instrumentos no nível ordinal (POWERS; XIE, 2000).

$\mathrm{Na}$ análise fatorial confirmatória, existe a premissa de normalidade das variáveis, principalmente a normalidade multivariada (HAIR et al., 2009). Autores como West, Finch e Curran (1995), Mulaik e James (1995), Barnes et al. (2001) reconhecem a dificuldade de se obter uma distribuição normal perfeita em pesquisas com escalas likert. Para avaliar essas questões, foram efetuados testes de normalidade (curtose e assimetria) das variáveis observáveis (BOLLEN, 1989). Constatou-se que algumas variáveis não apresentavam comportamento normal. Acrescenta-se ainda que a medida de curtose multivariada relativa foi de 1,440 , valor considerado relativamente baixo. Garson (2001) explica que, se o resultado for menor que 3,0, assume-se a existência de normalidade multivariada. Portanto, apesar de as variáveis observadas não demonstrarem normalidade univariada, coletivamente a distribuição multivariada é razoavelmente normal. Assim, no presente estudo, a opção foi seguir a sugestão de Barnes et al. (2001) de não transformar as variáveis não normalmente distribuídas, até porque esse procedimento pode provocar ainda mais problemas devido à distorção do verdadeiro significado das respostas (ANDERSON; GERBING, 1988; GASSENHEIMER; DAVIS; DAHLSTROM, 1998).

\subsubsection{Linearidade}

Hair et al. (2009) consideram prudente examinar todas as relações para identificar quaisquer desvios de linearidade que possam causar impacto na correlação. O modo mais usual de avaliar a linearidade é o exame do diagrama de dispersão das variáveis e identificar padrões não lineares nos dados. $\mathrm{O}$ exame desses diagramas revela alguns problemas de relações não lineares, especialmente com a variável observável ST 6, que foi mensurada com escala inversa, e as demais do construto Satisfação. Essa variável será eliminada na fase exploratória de análise do modelo de medida. As demais variáveis não apresentaram problemas de relações não lineares aparentes. Desse modo, transformações não foram consideradas necessárias.

\subsection{Análises das propriedades psicométricas}

A análise das propriedades psicométricas foi realizada, primeiramente, utilizando a análise fatorial exploratória (AFE) e, em seguida, a análise fatorial confirmatória (AFC).

\subsubsection{Análise fatorial exploratória}

Análise fatorial é uma técnica estatística que objetiva identificar a estrutura de um conjunto de variáveis, bem como fornecer um processo para redução de dados. Para Hair et al. (2009), no caso de se usarem escalas pré-existentes, o pesquisador deve verificar a unidimensionalidade de cada construto, isto é, confirmar, empiricamente, a presença das dimensões subjacentes que reflitam os construtos teoricamente apresentados.

\subsubsection{Suposições da análise fatorial}

A viabilidade da análise fatorial foi realizada, primeiramente, por análise visual das correlações, identificando os pares de variáveis que são estatisticamente significantes. A análise das matrizes de correlação revela que 106 das 108 correlações $(98 \%)$ são significativas no nível de 0,01. Em seguida, foi avaliada a significância geral da matriz de correlação por meio do teste de Bartlett. O resultado foi quiquadrado 4842; g1:325; Sig. 0,000. Esse resultado atesta a presença de correlações não nulas, porém, não garante o padrão linear dessas correlações.

Outra medida para avaliar o grau de inter-relações entre as variáveis e adequação da análise fatorial é a medida de adequação da amostra, também conhecida como KMO (Kaiser-Meyer-Olkin). Hair et al. (2009) e Malhotra (2001) recomendam 0,50 como valor mínimo para a estatística KMO. Neste estudo, o KMO foi igual 0,946 e encontra-se na faixa que justifica o uso da análise fatorial.

\subsubsection{Análise de componentes principais}

Foi utilizado o método de extração de fatores pela análise de componentes principais porque o objetivo era reduzir os dados para obtenção do mínimo número de fatores necessários para explicar o máximo de variância representado pelas variáveis originais. Optou-se pela rotação ortogonal, isto é, pela adoção do método VARIMAX, pois o objetivo era reduzir um número maior de variáveis para um conjunto menor de variáveis não correlacionadas. A análise das cargas rotacionadas, o teste de scree e o critério a priori contribuíram para a decisão de manter cinco fatores, que correspondem a uma variância explicada de, aproximadamente, $84 \%$ da variância das variáveis observadas. Foram consideradas as cargas fatoriais acima ou iguais a 0,35 (HAIR et al., 2009). A variável DR7 - "Eles são pró-ativos e informam se o pedido sofrerá algum atraso" - foi retirada, pois dividia carga entre os fatores 1 e 2 . Os resultados da AFE sugerem uma estrutura de cinco fatores, conforme mostra a Tabela 1.

Já havia a expectativa de que alguns itens da escala poderiam apresentar cargas fatoriais baixas e 
seriam eliminados para não prejudicar a validade e confiabilidade dos construtos. Os valores relativamente baixos de carregamentos nos respectivos fatores para os itens "DO1", "DO2", "DO9", "DR7", "ST6", "ST7", "CA1", "CC2", "CC4" e "CC6" implicaram a remoção dessas variáveis observáveis da análise. Os itens restantes reportam aos seus respectivos fatores com valores relativamente elevados de comunalidades e estatisticamente significativos, e correlacionam-se também de forma significativa com os outros itens do mesmo fator, conforme mostra a Tabela 1. Nota-se que nenhuma variável carrega significativamente em mais de um fator. De acordo com o significado implícito nos itens de cada fator, combinado com a literatura, o fator 1 foi designado como desempenho operacional; o fator 2, como desempenho relacional; o fator 3, como lealdade atitudinal; o fator 4, como satisfação; e o fator 5, como lealdade comportamental.

Para confirmar a confiabilidade interna, também foi feita a análise do alfa de Cronbach. O teste do coeficiente de Cronbach sugeriu a retenção de todos os itens nos seus respectivos fatores. Verifica-se, assim, a estrutura dimensional obtida por meio dos dados da AFE, sendo que os itens realmente "carregaram" em fatores comuns, que representam os construtos mencionados na teoria. Os construtos originais, testados pela Análise Fatorial Exploratória (AFE) e coeficiente de confiabilidade (alfa de Cronbach), sofreram algumas alterações. A Tabela 2 resume os resultados encontrados.

A AFE indicou a existência de cinco dimensões: DO, DR, ST, CA e CC, com cada conjunto de variáveis agora atuando como indicadores dos distintos construtos.

A AFE fornece uma indicação válida em termos de dimensionalidade, mas é insuficiente para garantir a unidimensionalidade, devido ao controle limitado do pesquisador sobre quais variáveis são indicadoras de qual construto latente. Um construto é considerado unidimensional, caso os indicadores utilizados para avaliar essa dimensão tenham somente um fator latente enquanto causa comum de suas variações,

Tabela 1. Matriz fatorial rotacionada VARIMAX de análise de componentes.

\begin{tabular}{|c|c|c|c|c|c|c|c|c|}
\hline \multirow[b]{3}{*}{ Variáveis } & \multicolumn{5}{|c|}{ Cargas Rotacionadas } & \multirow[b]{3}{*}{$\begin{array}{l}\text { Comuna- } \\
\text { lidade }\end{array}$} & \multirow[b]{3}{*}{$\begin{array}{l}\text { Alpha se } \\
\text { o item for } \\
\text { excluído }\end{array}$} & \multirow[b]{3}{*}{$\begin{array}{l}\text { Alpha de } \\
\text { Cronbach }\end{array}$} \\
\hline & \multicolumn{5}{|c|}{ VARIMAX } & & & \\
\hline & Fator 1 & Fator 2 & Fator 3 & Fator 4 & Fator 5 & & & \\
\hline DO3 & 0,81 & 0,28 & 0,21 & 0,27 & 0,14 & 0,87 & 0,949 & 0,960 \\
\hline DO4 & 0,80 & 0,29 & 0,07 & 0,27 & 0,20 & 0,84 & 0,954 & \\
\hline DO5 & 0,83 & 0,26 & 0,24 & 0,21 & 0,16 & 0,89 & 0,948 & \\
\hline DO6 & 0,77 & 0,33 & 0,25 & 0,26 & 0,11 & 0,84 & 0,951 & \\
\hline DO7 & 0,77 & 0,35 & 0,18 & 0,20 & 0,17 & 0,82 & 0,953 & \\
\hline DO8 & 0,78 & 0,33 & 0,21 & 0,13 & 0,09 & 0,79 & 0,956 & \\
\hline DR1 & 0,32 & 0,70 & 0,24 & 0,33 & 0,28 & 0,84 & 0,945 & 0,955 \\
\hline DR2 & 0,42 & 0,74 & 0,28 & 0,27 & 0,09 & 0,89 & 0,942 & \\
\hline DR3 & 0,43 & 0,75 & 0,26 & 0,28 & 0,08 & 0,90 & 0,941 & \\
\hline DR4 & 0,43 & 0,70 & 0,27 & 0,18 & 0,09 & 0,79 & 0,952 & \\
\hline DR5 & 0,38 & 0,69 & 0,27 & 0,25 & 0,27 & 0,83 & 0,945 & \\
\hline DR6 & 0,36 & 0,61 & 0,30 & 0,33 & 0,17 & 0,72 & 0,954 & \\
\hline ST1 & 0,41 & 0,46 & 0,35 & 0,60 & 0,11 & 0,87 & 0,936 & 0,954 \\
\hline ST2 & 0,41 & 0,44 & 0,37 & 0,61 & 0,13 & 0,88 & 0,935 & \\
\hline ST3 & 0,41 & 0,44 & 0,38 & 0,61 & 0,12 & 0,90 & 0,934 & \\
\hline ST4 & 0,37 & 0,33 & 0,15 & 0,70 & 0,24 & 0,81 & 0,960 & \\
\hline ST5 & 0,40 & 0,37 & 0,36 & 0,62 & 0,06 & 0,82 & 0,949 & \\
\hline LA2 & 0,23 & 0,35 & 0,72 & 0,17 & 0,30 & 0,81 & 0,941 & 0,950 \\
\hline LA3 & 0,26 & 0,31 & 0,75 & 0,12 & 0,35 & 0,85 & 0,937 & \\
\hline LA4 & 0,20 & 0,20 & 0,80 & 0,17 & 0,27 & 0,82 & 0,941 & \\
\hline LA5 & 0,20 & 0,31 & 0,80 & 0,25 & 0,22 & 0,89 & 0,932 & \\
\hline LA6 & 0,17 & 0,15 & 0,85 & 0,18 & 0,22 & 0,86 & 0,940 & \\
\hline LC1 & 0,21 & 0,13 & 0,31 & 0,16 & 0,81 & 0,84 & 0,882 & 0,906 \\
\hline LC3 & 0,11 & 0,13 & 0,18 & 0,05 & 0,92 & 0,91 & 0,823 & \\
\hline LC5 & 0,14 & 0,12 & 0,36 & 0,09 & 0,80 & 0,81 & 0,890 & \\
\hline
\end{tabular}


ou seja, caso essas medidas avaliem somente um conceito teórico. A próxima etapa - análise fatorial confirmatória - tem a função de validar o resultado da análise fatorial.

\subsubsection{Análise fatorial confirmatória}

Neste estudo, os ajustes dos cinco construtos requeridos foram avaliados de forma isolada, para melhor compreensão das relações entre as variáveis, em especial a análise de validade discriminante. Dessa forma, cinco análises fatoriais confirmatórias (AFC) foram realizadas. Os resultados finais estão reproduzidos na Tabela 3 .

Os resultados da AFC inicial demonstraram que um indicador da dimensão satisfação estava comprometendo o ajuste do modelo, pois reportava uma baixa carga padronizada do coeficiente de explicação $\left(\mathrm{R}^{2}\right)$ com o seu respectivo construto. Como decisão, o indicador ST4 "Eles fornecem completamente todos os serviços que eu quero deles" foi suprimido. Pode ter ocorrido algum problema de interpretação desta afirmativa por parte dos respondentes.

Porém, mesmo assim, o número restante de indicadores foi suficiente para a manutenção da confiabilidade dos construtos estudados, uma vez que três é o número mínimo de indicadores exigido para compor uma variável latente (NUNNALLY; BERNSTEIN, 1994). Desse modo, a versão final do questionário ficou com 24 indicadores, contra os 35 propostos inicialmente.

Para utilizar as estimativas padronizadas com mais segurança, foi avaliada a qualidade do ajuste obtido. Ao examinar os resultados, não foram encontrados variáveis com erro de mensuração negativos, coeficientes padronizados excedentes ou muito próximos de um, e nem erros padrão elevados, logo, pôde-se prosseguir a avaliação da qualidade de ajuste da análise fatorial confirmatória.

Os resultados da qualidade de ajuste são mostrados na Tabela 4.

Para avaliar o ajuste do modelo de mensuração proposto, três índices foram avaliados (absoluto, incremental e parcimonioso). Os dados da Tabela 4 fornecem evidências de que o ajuste do modelo foi satisfatório, apesar de algumas medidas estarem fora dos limites recomendados. Esse é o caso do quiquadrado, que deveria ser não significativo, mas mostrou-se significativo em alguns casos. Esse resultado era esperado, pois as estatísticas baseadas no quiquadrado costumam produzir tais resultados para amostras < 200 (HAIR et al., 2009), como é o caso da amostra utilizada nesta pesquisa. Assim, Raykov e Marcoulides (2000) recomendam que outros índices de ajustamento devem ser analisados, para que se possa ter uma ideia real do ajuste do modelo. O GFI de 0,99 e o RMSEA abaixo de 0,08 indicam que o ajuste absoluto foi aceitável, já que essas medidas estão dentro dos limites recomendados. Isso significa que os construtos preveem adequadamente a matriz de correlação observada. Em relação ao ajuste incremental, o AGFI situou-se entre 0,92 e 0,97 e o NNFI entre 0,99 e 1,00, acima do mínimo recomendado, que é de 0,90 (HAIR et al., 2009). Esses resultados indicam que o modelo estimado é superior ao modelo nulo, no qual os valores da matriz de covariâncias entre as variáveis latentes são iguais a zero. $\mathrm{O}$ ajuste parcimonioso diz respeito à relação entre a qualidade de ajuste do modelo estimado com o número de coeficientes exigidos para atingir um nível adequado de ajuste, sendo recomendável que as medidas estejam o mais próximo possível de 1. Isso ocorreu com aquelas incluídas na análise, pois seus

Tabela 2. Resultado da Análise Fatorial Exploratória.

\begin{tabular}{|c|c|c|c|c|}
\hline Construto & $\begin{array}{c}\text { Modelo teórico } \\
\text { proposto }\end{array}$ & $\begin{array}{l}\text { Indicadores } \\
\text { excluídos }\end{array}$ & $\begin{array}{l}\text { Total final de } \\
\text { indicadores }\end{array}$ & Resultado encontrado \\
\hline $\begin{array}{l}\text { Desempenho operacional } \\
\text { (DO) }\end{array}$ & $\begin{array}{l}1 \text { Dimensão } \\
9 \text { indicadores } \\
\text { Escala Likert } 7 \text { pontos }\end{array}$ & 3 & 6 & $\begin{array}{l}\text { Confirmada a unidimensionalidade } \\
\text { do construto e confiabilidade da } \\
\text { escala }\end{array}$ \\
\hline $\begin{array}{l}\text { Desempenho relacional } \\
\text { (DR) }\end{array}$ & $\begin{array}{l}1 \text { Dimensão } \\
7 \text { indicadores } \\
\text { Escala Likert } 7 \text { pontos }\end{array}$ & 1 & 6 & $\begin{array}{l}\text { Confirmada a unidimensionalidade } \\
\text { do construto e confiabilidade da } \\
\text { escala }\end{array}$ \\
\hline Satisfação (ST) & $\begin{array}{l}1 \text { Dimensão } \\
7 \text { indicadores } \\
\text { Escala Likert } 7 \text { pontos }\end{array}$ & 2 & 5 & $\begin{array}{l}\text { Confirmada a unidimensionalidade } \\
\text { do construto e confiabilidade da } \\
\text { escala }\end{array}$ \\
\hline Lealdade atitudinal(CA) & $\begin{array}{l}1 \text { Dimensão } \\
6 \text { indicadores } \\
\text { Escala Likert } 7 \text { pontos }\end{array}$ & 1 & 5 & $\begin{array}{l}\text { Confirmada a unidimensionalidade } \\
\text { do construto e confiabilidade da } \\
\text { escala }\end{array}$ \\
\hline $\begin{array}{l}\text { Lealdade } \\
\text { comportamental (CC) }\end{array}$ & $\begin{array}{l}1 \text { Dimensão } \\
6 \text { indicadores } \\
\text { Escala Likert } 7 \text { pontos }\end{array}$ & 3 & 3 & $\begin{array}{l}\text { Confirmada a unidimensionalidade } \\
\text { do construto e confiabilidade da } \\
\text { escala }\end{array}$ \\
\hline
\end{tabular}


valores CFI foram iguais a 1,0. Em resumo, as várias medidas de qualidade de ajuste geral fornecem apoio suficiente para considerar os resultados como uma representação aceitável dos construtos teorizados.

Depois da análise destes ajustes globais, deve-se atentar aos ajustes específicos do modelo de mensuração, quanto à unidimensionalidade e à confiabilidade. A unidimensionalidade é a característica de um conjunto de indicadores que tem apenas um traço inerente ou conceito em comum (HAIR et al., 2009). Avaliar a unidimensionalidade consiste em verificar se os indicadores estabelecidos representam um único construto, procedimento que já foi executado na AFE. Uma medida aceitável da unidimensionalidade também deverá indicar baixos resíduos padronizados. Segundo Garver e Mentzer (1999) e Jöreskog e Sörbom (1993), para que uma dimensão possua unidimensionalidade, é necessário que todos os seus resíduos padronizados sejam inferiores a 2,58. A maior carga residual padronizada encontrada no estudo foi de 2,34 na dimensão lealdade atitudinal, podendo-se, assim, conferir unidimensionalidade a todas as dimensões da escala. Outra medida indicadora de unidimensionalidade é o

Tabela 3. Resultado da Análise Fatorial Confirmatória.

\begin{tabular}{cccccc}
\hline Variável latente & $\begin{array}{c}\text { Variável } \\
\text { observável }\end{array}$ & $\begin{array}{c}\text { Carga } \\
\text { padronizada }\end{array}$ & $\mathbf{t}$ & $\mathbf{p}$ & $\begin{array}{c}\mathbf{R}^{\mathbf{2}} \\
\text { dos itens }\end{array}$ \\
\hline & DO3 & 0,91 & - & - & 0,83 \\
& DO4 & 0,86 & 19,42 & 0,050 & 0,75 \\
Desempenho Operacional & DO5 & 0,95 & 20,52 & 0,048 & 0,90 \\
& DO6 & 0,91 & 18,39 & 0,055 & 0,83 \\
& DO7 & 0,85 & 15,46 & 0,060 & 0,73 \\
& DO8 & 0,83 & 14,35 & 0,059 & 0,68 \\
\hline \multirow{5}{*}{ Desempenho Relacional } & DR1 & 0,89 & - & - & 0,79 \\
& DR2 & 0,95 & 19,01 & 0,061 & 0,90 \\
& DR3 & 0,95 & 19,21 & 0,060 & 0,90 \\
& DR4 & 0,83 & 13,96 & 0,071 & 0,69 \\
& DR5 & 0,83 & 17,35 & 0,054 & 0,69 \\
& DR6 & 0,79 & 12,60 & 0,072 & 0,62 \\
\hline \multirow{5}{*}{ Satisfação } & ST1 & 0,97 & - & - & 0,94 \\
& ST2 & 0,96 & 30,95 & 0,032 & 0,93 \\
& ST3 & 0,96 & 29,71 & 0,035 & 0,92 \\
& ST5 & 0,82 & 16,00 & 0,055 & 0,67 \\
\hline \multirow{5}{*}{ Comprometimento afetivo } & CA2 & 0,85 & - & - & 0,72 \\
& CA3 & 0,86 & 19,54 & 0,053 & 0,74 \\
& CA4 & 0,89 & 13,27 & 0,083 & 0,79 \\
& CA5 & 0,93 & 15,38 & 0,074 & 0,87 \\
\hline \multirow{5}{*}{ Lealdade comportamental } & CA6 & 0,90 & 14,56 & 0,084 & 0,81 \\
\hline & CC1 & 0,85 & - & - & 0,72 \\
& CC3 & 0,95 & 14,42 & 0,083 & 0,90 \\
& CC5 & 0,84 & 12,87 & 0,096 & 0,71 \\
\hline
\end{tabular}

Tabela 4. Modelos de mensuração dos construtos desempenho relacional, desempenho operacional, satisfação, lealdade atitudinal e lealdade comportamental.

\begin{tabular}{lcccccc}
\hline \multicolumn{1}{c}{ Tipo de ajuste } & \multicolumn{3}{c}{ Absoluto } & \multicolumn{2}{c}{ Incremental } & Parcimo-nioso \\
\hline \multicolumn{1}{c}{ Construto } & $\mathbf{X}^{2} / g l$ & RMSEA & GFI & AGFI & NNFI & CFI \\
\hline Desempenho Relacional & 0,544 & 0,000 & 0,99 & 0,97 & 1,00 & 1,00 \\
Desempenho Operacional & 0,838 & 0,000 & 0,99 & 0,96 & 1,00 & 1,00 \\
Satisfação & 1,82 & 0,074 & 0,99 & 0,94 & 0,99 & 1,00 \\
Lealdade Atitudinal & 1,89 & 0,078 & 0,99 & 0,92 & 0,99 & 1,00 \\
Lealdade comportamental & - & 0,000 & $*$ & $*$ & $*$ & $*$ \\
\hline
\end{tabular}

*Ajustamento perfeito. 
exame das cargas indicadoras quanto à significância estatística. Para cada variável, a Tabela 3 mostra que os valores associados com cada uma das cargas excedem os valores críticos para o nível de significância de 0,05 (valor crítico = 2,56). Logo, todas as variáveis observáveis estão significantemente relacionadas com suas variáveis latentes especificadas.

Também foram utilizados os valores da confiabilidade composta e variância extraída para a mensuração da confiabilidade. Pode-se usar a seguinte fórmula para calcular a confiabilidade composta do construto:

\section{Confiabilidade (composta) do construto $=\left(\Sigma \lambda_{j}\right)^{2} /\left[\left(\Sigma \lambda_{\mathrm{j}}\right)^{2}+\Sigma \lambda_{j}\right]$}

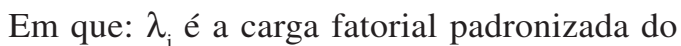
indicador $j$ e $E_{j}$ é o erro de mensuração do indicador $j$, calculado como 1- confiabilidade do indicador.

Segundo Hair et al. (2009), são aceitos valores iguais ou superiores a 0,70 para a confiabilidade composta. Valores abaixo de 0,70 provocam a exclusão do item. Uma medida complementar da confiabilidade é a medida da variância extraída. Ela reflete a quantidade total de variância dos indicadores explicada pela variável latente. A medida da variância extraída pode ser calculada pela seguinte fórmula:

Variância extraída $=\left(\Sigma \lambda_{\mathrm{j}}^{2}\right) /\left[\left(\Sigma \lambda_{\mathrm{j}}^{2}\right)+\Sigma E_{j}\right]$

Em que: $\lambda \mathrm{j}$ é a carga fatorial padronizada do indicador $j$ e $E j$ é o erro de mensuração do indicador $j$, calculado como 1- confiabilidade do indicador.

A diferença entre a confiabilidade composta do construto e a medida da variância extraída é o fato de que, nesta última, as cargas padronizadas são elevadas ao quadrado antes de serem somadas. Bons valores para um construto devem ser iguais ou acima de 0,5 (GARVER; MENTZER, 1999; HAIR et al., 2009). Assim, a Tabela 5 apresenta os valores da confiabilidade composta e variância extraída para todas as dimensões do construto, aferindo a sua confiabilidade.

Todos os construtos excedem o nível recomendado de 0,70 para a confiabilidade composta e superam a medida de 0,50 para variância extraída. Portanto, pode-se concluir que há evidências de que as medidas utilizadas são confiáveis.

\subsubsection{Validade}

Depois do estabelecimento da unidimensionalidade e da confiabilidade, a validade do construto deve ser investigada. A validade convergente avalia se os indicadores designados para a variável latente estatisticamente convergem. Dunn, Seaker e Waller (1994) afirmam que, se as cargas individuais dos indicadores que representam uma variável latente são estatisticamente significantes, então se pode inferir a existência de validade convergente. A significância das medidas individuais dos indicadores pode ser dada pelo exame dos $t$-valor, sendo que valores acima de |1,96| são considerados significantes em nível de 0,05 (BAGOZZI; YI; PHILLIPS, 1991). Hair et al. (2009) afirmam ainda a necessidade da correlação entre cada indicador e a variável latente ser igual ou superior a 0,50 para a aferição da validade convergente. Ambos os pré-requisitos foram levados em consideração neste estudo e, como pode ser observado na Tabela 6 , as especificações para a confirmação da validade convergente foram atingidas.

Entre os vários métodos para estabelecer a validade discriminante, Fornell e Larcker (1981) recomendam a comparação da variância extraída de cada construto com as variâncias compartilhadas (o quadrado do coeficiente de correlação) entre os construtos. Desta forma, é observado que o valor da variância compartilhada é menor do que o da variância extraída na maioria dos construtos. Para os construtos estudados, foi verificada a validade discriminante entre todas as dimensões, conforme pode ser observado na Tabela 7, quando a variância extraída (destacada na diagonal em negrito) é percebida como maior que a variância compartilhada entre as dimensões.

Nota-se, na Tabela 7, que nenhum coeficiente de determinação teve valor superior ao da variância extraída de cada construto. Portanto, os dados indicam que a validade discriminante foi atestada para o modelo.

Tabela 5. Resultados da análise de confiabilidade.

\begin{tabular}{lcc}
\hline \multicolumn{1}{c}{ Variável latente } & $\begin{array}{c}\text { Confiabilidade } \\
\text { Confiabilidade } \\
\text { Composta }\end{array}$ & $\begin{array}{c}\text { Variância } \\
\text { extraída }\end{array}$ \\
\hline $\begin{array}{l}\text { Desempenho } \\
\text { Operacional }\end{array}$ & 0,96 & 0,79 \\
\hline $\begin{array}{l}\text { Desempenho } \\
\text { Relacional }\end{array}$ & 0,95 & 0,77 \\
\hline Satisfação & 0,96 & 0,86 \\
\hline $\begin{array}{l}\text { Comprometimento } \\
\text { afetivo }\end{array}$ & 0,95 & 0,79 \\
\hline $\begin{array}{l}\text { Lealdade } \\
\text { comportamental }\end{array}$ & 0,91 & 0,93 \\
\hline
\end{tabular}

Tabela 6. Resultados da análise de validade.

\begin{tabular}{lcc}
\hline \multicolumn{1}{c}{ Variável latente } & $\begin{array}{c}\text { Validade Convergente } \\
\text { Menor carga } \\
\text { fatorial } \\
\text { padronizada }\end{array}$ & $\begin{array}{c}\text { Menor } \\
\text { t-valor }\end{array}$ \\
\hline Desempenho Operacional & 0,83 & 14,35 \\
Desempenho Relacional & 0,79 & 12,60 \\
Satisfação & 0,82 & 16,00 \\
Comprometimento afetivo & 0,86 & 13,27 \\
Lealdade comportamental & 0,84 & 12,87 \\
\hline
\end{tabular}


Tabela 7. Indicadores de validade discriminante.

\begin{tabular}{lccccc}
\hline & $\begin{array}{c}\text { Desempenho } \\
\text { relacional }\end{array}$ & $\begin{array}{c}\text { Desempenho } \\
\text { operacional }\end{array}$ & Satisfação & $\begin{array}{c}\text { Lealdade } \\
\text { Atitudinal }\end{array}$ & $\begin{array}{c}\text { Lealdade } \\
\text { comportamental }\end{array}$ \\
\hline Desempenho relacional & $\mathbf{0 , 7 9 *}$ & & & & \\
Desempenho operacional & 0,69 & $\mathbf{0 , 7 7 ^ { * }}$ & & & \\
Satisfação & 0,77 & 0,66 & $\mathbf{0 , 8 6 *}$ & & \\
Lealdade Atitudinal & 0,52 & 0,41 & 0,58 & $\mathbf{0 , 7 9 *}$ & \\
Lealdade comportamental & 0,20 & 0,16 & 0,22 & 0,41 & $\mathbf{0 , 9 3 *}$ \\
\hline *Variância extraída. & & & & &
\end{tabular}

Tabela 8. Indicadores de validade discriminante.

\begin{tabular}{lccccc}
\hline & $\begin{array}{c}\text { Desempenho } \\
\text { relacional }\end{array}$ & $\begin{array}{c}\text { Desempenho } \\
\text { operacional }\end{array}$ & Satisfação & $\begin{array}{c}\text { Lealdade } \\
\text { Atitudinal }\end{array}$ & $\begin{array}{c}\text { Lealdade } \\
\text { comportamental }\end{array}$ \\
\hline Desempenho relacional & 1,00 & & & & \\
Desempenho operacional & $0,83^{*}$ & 1,00 & & & \\
Satisfação & $0,88^{*}$ & $0,81^{*}$ & 1,00 & & \\
Lealdade Atitudinal & $0,72^{*}$ & $0,64^{*}$ & $0,76^{*}$ & 1,00 & \\
Lealdade comportamental & $0,45^{*}$ & $0,40^{*}$ & $0,47^{*}$ & $0,64^{*}$ & 1,00 \\
\hline
\end{tabular}

*Correlação significativa a 0,01 .

Como forma de complementar esta análise, foram efetuadas as correlações entre as variáveis latentes do modelo. Observou-se que não foram constatados valores superiores a 0,95 , o que indica que as correlações convergem para cada construto analisado e são diferentes entre si. Os resultados são mostrados pela Tabela 8 .

Diante desses resultados, pode-se concluir que as estimativas padronizadas do modelo de relacionamento cliente-fornecedor podem ser consideradas como válidas. Essa conclusão é reforçada pelo fato de a matriz de resíduos padronizados do modelo ter apresentado menos de $6,7 \%$ de valores fora do intervalo recomendado por Hair et al. (2009), que é de $[-2,58 ; 2,58]$.

A percepção da existência de validade discriminante para as variáveis latentes estudadas, juntamente com a confirmação da existência dos ajustes favoráveis dos modelos de unidimensionalidade, de confiabilidade e de validade convergente confirmam o processo de aferição de qualidade das escalas propostas.

\section{Conclusões e limitações}

Nos últimos anos, a literatura de Supply Chain Management tem se tornado essencial na área da Gestão de Produção e Operações e muitos instrumentos para a sua avaliação têm sido desenvolvidos, a grande parte em inglês. Existem poucas medidas específicas confiáveis e válidas adaptadas à cultura brasileira, disponíveis para a avaliação dos construtos serviço ao cliente, satisfação e lealdade. Por isso, no presente estudo, as recomendações preconizadas na literatura para adaptação cultural e validação desses construtos foram seguidas.
Os resultados dos coeficientes alpha de Cronbach, a AFE e AFC obtidos nos construtos de serviço ao cliente, satisfação e lealdade favoreceram a manutenção de 24 dos 35 itens originais. As exclusões de três itens do construto de desempenho operacional, um item de desempenho relacional, dois itens de satisfação, um item de lealdade atitudinal e três itens de lealdade comportamental que não carregaram adequadamente as cargas desejadas, aumentaram o alpha de Cronbach de cada construto. A análise da consistência interna dos construtos originais resultou no coeficiente alpha de Cronbach de 0,960; 0,955; 0,954; 0,950; e 0,906 para os domínios de desempenho operacional, desempenho relacional, satisfação, lealdade atitudinal e lealdade comportamental, respectivamente.

A análise da validade de conteúdo indicou que a concordância entre os especialistas não foi unânime, devido a algumas respostas discordantes de um especialista. Assim, os itens foram revistos e, finalmente, considerados adequados para a confirmação da validade de conteúdo dos construtos.

A análise fatorial foi realizada para determinar a pertinência dos itens em cada construto. Os resultados obtidos mostram que itens investigados correspondem aos cinco construtos esperados. Apenas 11 dos 35 itens estiveram locados nas componentes principais. Esse resultado é adequado, pois pesquisa similar na cultura americana, com os mesmos construtos, apresentaram resultados parecidos (DAVIS-SRAMEK; MENTZER; STANK, 2008).

As correlações positivas e significativas entre o par de construtos desempenho relacional e desempenho operacional $(\mathrm{r}=0,83)$ foram coerentes em relação ao reportado por outros autores (STANK; 
GOLDSBY; VICKERY, 1999; STANK et al., 2003; DAVIS-SRAMEK; MENTZER; STANK, 2008). Esse escore confirma que o relacionamento pessoal entre vendedores e compradores é importante, podendo inclusive ajudar a resolver problemas no nível operacional sem afetar a satisfação do comprador. Resultados similares foram encontrados entre desempenho operacional e satisfação $(r=0,81)$, conforme previsto por Daugherty, Stank e Ellinger (1998) e Leuthesser e Kohli (1995); satisfação e lealdade atitudinal $(r=0,76)$, conforme estimado por Fornell (1992) e Reichheld e Sasser (1990); lealdade atitudinal e lealdade comportamental $(r=0,64)$, reportado por Dick e Basu (1994) e Davis-Sramek, Mentzer e Stank (2008). Estes resultados indicam que os construtos estão relacionados. A correlação positiva entre os construtos demonstrou que eles não são independentes, isto é, quanto melhor o nível de serviço, maior a satisfação e maior a lealdade do cliente.

As análises dos construtos demonstraram boas propriedades psicométricas em termos de confiabilidade e validade. É importante ressaltar que a validação de um instrumento não pode ser esgotada em um único estudo (HAIR et al., 2009). Portanto, estudos posteriores são necessários, considerando que este é o primeiro estudo que avalia estes construtos específicos em português. A análise iniciou com 35 itens possíveis e concluiu com 24 itens considerados válidos, ou seja, $69,0 \%$ dos itens foram validados.

Os itens que formaram os construtos pesquisados foram apresentados para cinco profissionais da área de compras e qualitativamente considerados importantes na relação cliente-fornecedores, principalmente o desempenho relacional, pouco mencionada na literatura, mas muito importante para os compradores avaliarem seus fornecedores, pois, raramente, existem notícias do desempenho operacional do fornecedor, somente em casos de emergências são notificados em suas organizações.

Como qualquer estudo, este possui suas limitações. Uma primeira é a apuração da percepção de apenas uma pessoa (o comprador) que continua sendo uma opinião individual por mais competente que seja.

A segunda limitação é a violação da normalidade multivariada, mas esta prática é usual em investigações da área de ciências sociais.

Uma terceira limitação é a amostra colhida por conveniência e restrita a uma indústria (empresas químicas) e a uma categoria de produtos (itens de alavancagem), inviabilizando a generalização dos resultados para todos os mercados. Mesmo a população de interesse tendo sido definida como comprador da indústria química, é possível existir um viés oriundo da coleta de dados somente de compradores de grandes empresas.
Uma sugestão de pesquisa futura seria a aplicação deste estudo em outros setores da economia brasileira (considerando o cuidado de buscar amostras maiores) e a comparação dos resultados encontrados entre os setores. Mesmo diante dessa necessidade, este estudo tem implicações teóricas no âmbito do relacionamento cliente-fornecedor, além de auxiliar no esforço de descrição do fenômeno em questão.

\section{Referências}

ALVES, L. R. R.; BORNIA, A. C. Desenvolvimento de uma escala para medir o potencial empreendedor utilizando a Teoria da Resposta ao Item (TRI). Gestão \& Produção, v. 18, n. 4, 2011. http://dx.doi.org/10.1590/ S0104-530X2011000400007

ANDERSON, J. C.; GERBING, D. W. Structural Equation Modeling in Practice: A Review and Recommended Two-Step Approach. Psychological Bulletin, v. 103, n. 3, 411-423, 1988. http://dx.doi. org/10.1037/0033-2909.103.3.411

ANDERSON, E. W.; FORNELL, C.; LEHMANN, D. R. Customer Satisfaction, Market Share, and Profitability: Findings from Sweden. Journal of Marketing, v. 58, n. 3, p. 53-67, 1994. http://dx.doi.org/10.2307/1252310

BAGOZZI, R. P.; YI, Y.; PHILLIPS, W. L. Assessing construct validity in organizational research. Administrative Science Quarterly, v. 36, n. 3, p. 421-458, Sept 1991. http://dx.doi.org/10.2307/2393203

BARNES, J. et al. Factor analysis: checking assumptions of normality before conducting factor analysis. Journal of consumer psychology, v. 10, n. 1-2, p. 79-81, 2001.

BAUMGARTNER, H.; HOMBURG, C. Applications of structural equation modeling in marketing and consumer research. International Journal of Research in Marketing, v. 13, p. 139-161, 1996. http://dx.doi. org/10.1016/0167-8116(95)00038-0

BOLLEN, K. A. Structural equations with latent variables. New York: Wiley, 1989.

BRANCO, G. M.; RIBEIRO, J. L. D.; TINOCO, M. A. C. Determinantes da satisfação e atributos da qualidade em serviços de hotelaria. Produção, v. 20, n. 4, p. 576-588, 2010. http://dx.doi.org/10.1590/ S0103-65132010005000057

CARUANA, A. Service loyalty: the effects of service quality and the mediating role of customer satisfaction. European Journal of Marketing, v. 36, n. 7-8, p. 811-829, 2002. http://dx.doi.org/10.1108/03090560210430818

CHA, E.; KIM, K. H.; ERLEN, J. A. Translation of scales in cross-cultural research: issues and techniques. Journal of Advanced Nursing, v. 58, n. 4, p. 386-395, 2007. http://dx.doi.org/10.1111/j.1365-2648.2007.04242.x

CHANDHURI, A.; HOLBROOK, M. B. The chain of effects from brand trust and brand affect to brand performance: the role of brand loyalty. Journal of Marketing, v. 65, n. 2, p. 81-93, 2001. http://dx.doi. org/10.1509/jmkg.65.2.81.18255

COLLIER, D. A. A service quality process map for credit card processing. Decision Sciences, v. 22, n. 2, p. 406-420, 1991. http://dx.doi. org/10.1111/j.1540-5915.1991.tb00355.x

CRAIG, C. S.; DOUGLAS, S. P. Beyond national culture: Implications of cultural dynamics for consumer research. 
International Marketing Review, v. 23, n. 3, p. 322-342, 2006. http://dx.doi.org/10.1108/02651330610670479

DAVIS, E. R. The role of logistics service quality in creating customer loyalty. 2006. Dissertation (Master's degree)-University of Tennessee, Knoxville, 2006.

DAVIS-SRAMEK, B.; MENTZER, J. T.; STANK, T. P. Creating consumer durable retailer customer loyalty through order fulfillment service operations. Journal of Operations Management, v. 26, p. 781-797, 2008. http://dx.doi.org/10.1016/j.jom.2007.07.001

DAVIS-SRAMEK, B. et al. Creating commitment and loyalty behavior among retailers: what are the roles of service quality and satisfaction? Journal of the Academy of Marketing Science, v. 37, p. 440-454, 2009. http:// dx.doi.org/10.1007/s11747-009-0148-y

DAUGHERTY, P. J.; STANK, T. P.; ELLINGER, A. E. Leveraging logistics/distribution capabilities: the effect of logistics service on market share. Journal of Business Logistics, v. 19, n. 2, p. 35-52, 1998.

DICK, A. S.; BASU, K. Customer loyalty: toward an integrated conceptual framework. Journal of the Academy of Marketing Science, v. 22, n. 2, p. 99-113, 1994. http:// dx.doi.org/10.1177/0092070394222001

DILLMAN, D. A. Mail and Internet surveys: The tailored design method. 2nd ed. New York: John Wiley \& Sons, Inc.; 2000.

DUNN, S. C.; SEAKER, R. F.; WALLER, M. A. Latent variable in business logistics research: scale development and validation. Journal of Business Logistics, v. 15, n. 2, p. 145-173, 1994.

FLEURY, P. F.; SILVA, C. R. L. Avaliação do serviço de distribuição física: a relação entre a indústria de bens de consumo e o comércio atacadista e varejista. Gestão \& Produção, v. 4, n. 2, p. 204-218, ago. 1997.

FORNELL, C. A National Customer Satisfaction Barometer: the Swedish experience. Journal of Marketing, v. 56, n. 1, p. 6-21, 1992. http://dx.doi.org/10.2307/1252129

FORNELL, C.; LARCKER, D. F. Evaluating Structural Equation Models with Unobservable Variables and Measurement Error. Journal of Marketing Research, v. 18, n. 1, p. 39-50, 1981. http://dx.doi. org/10.2307/3151312

GARBARINO, E.; JOHNSON, M. The different roles of satisfaction, trust, and commitment in customer relationships. Journal of Marketing, v. 63, n. 2, p. 70-87, 1999. http://dx.doi.org/10.2307/1251946

GARSON, D. PA 765 - Quantitative research in public administration: 2001. 2001. Disponível em <http:// www2.chass.ncsu.edu/garson/pa765/logistic.htm>. Acesso em: 01 fev. 2012.

GARVER, M. S.; MENTZER, J. T. Logistics research methods: Employing structural equation modeling to test for construct validity. Journal of Business Logistics, v. 20, n. 1, 1999.

GASSENHEIMER, J.; DAVIS, J. D.; DAHLSTROM, R. Is dependent what we want to be? Journal of Retailing, v. 74, n. 2, 247-71, 1998. http://dx.doi.org/10.1016/ S0022-4359(99)80095-5

GRÖNROOS, C. A service quality model and its marketing implications. European Journal of Marketing. v. 18, n. 4, p. 36-44, 1984. http://dx.doi.org/10.1108/ EUM0000000004784

HAIR, J. F. et al. Multivariate data analysis. 6th ed. Englewood Cliffs: Prentice Hall, 2009.
HILL, M. M.; HILL, A. Investigação por Questionário. Lisboa: Silabo, 2000.

JÖRESKOG, K.; SÖRBOM, D. Lisrel 8: structural equations with the SIMPLIS command language. New Jersey: Lawrence Erlbaum Associates, 1993.

KANDAMPULLY, J. Service Quality to Service Loyalty: a Relationship Which Goes Beyond Customer Services, Total Quality Management, v. 9, n. 6, p. 431-443, 1998. http://dx.doi.org/10.1080/0954412988370

KEININGHAM, T. et al. The value of different customer satisfaction and loyalty metrics in predicting customer retention, recommendation, and share-of-wallet. In: Managing Service Quality, v. 17, n. 4, p. 361-84, 2007. http://dx.doi.org/10.1108/09604520710760526

KIM, K.; FRAZIER, G. L. Measurement of distributor commitment in industrial channels of distribution. Journal of Business Research, v. 40, n. 2, p. 139-154, 1997. http://dx.doi.org/10.1016/S0148-2963(96)00284-6

KINNEAR, T.; TAYLOR, J. Marketing research: an applied approach. New York: McGraw-Hill, 1991.

KRALJIC, P. Purchasing Must Become Supply Management. Harvard Business Review, n. 83509, p.109-117, 1983.

KUMAR, N.; HIBBARD, J. D.; STERN, L. W. An Empirical Assessment of the Nature and Consequences of Marketing Channel Intermediary Commitment. Cambridge: Marketing Science Institute, 1994.

KUMAR, V.; BOHLING, T. R.; LADDA, R. N. Antecedents and consequences of the relationship intention: implications for transaction and relationship marketing. Industrial Marketing Management, v. 32, n. 8, p. 667-677, 2003.

LAMBERT, D. M.; COOPER, M. C.; PAGH, J. D. Supply chain management: Implementation issues and research opportunities. The International Journal of Logistics Management, v. 9, n. 2, 1998. http://dx.doi. org/10.1108/09574099810805807

LARÁN, J. A.; ESPINOZA, F. S. Consumidores satisfeitos, e então? Analisando a satisfação como antecedente da lealdade. Revista de Administração Contemporânea, v. 8, n. 2, p. 51-70, 2004. http://dx.doi.org/10.1590/ S1415-65552004000200004

LEUTHESSER, L.; KOHLI, A. K. Relational behavior in business markets. Journal of Business Research, v. 34, p. 221-33, 1995. http://dx.doi. org/10.1016/0148-2963(95)00006-E

MALHOTRA, N. K. Pesquisa de marketing: uma orientação aplicada. 3. ed. Porto Alegre: Bookman, 2001. 719 p.

MATILLA, A. S. The impact of relationship type on customer loyalty in a context of service failures. Journal of Service Research, v. 4, n. 2, p. 91-101, 2001. http:// dx.doi.org/10.1177/109467050142002

MENTZER, J. T.; FLINT, D. J.; HULT, G. T. M. Logistics service quality as a segment-customized process. Journal of Marketing, v. 65, n. 2, p. 82-104, 2001. http://dx.doi. org/10.1509/jmkg.65.4.82.18390

MULAIK, S. A.; JAMES, L. R. Objectivity and reasoning in Science and Structural Equation Modeling. In: HOYLE, R. H. (Ed.). Structural Equation Modeling: concepts, issues and applications. London: Sage Publications Inc., 1995. cap. 7, p. 118-137.

NOVATO, T. S.; GROSSI, S. A.; KIMURA, M. Cultural adaptation and validation of the "Diabetes Quality of Life for Youths" measure of Ingersoll and Marrero 
into Brazilian culture. Revista Latino-Americana de Enfermagem, v. 16, n. 2, p. 224-30, 2008. http://dx.doi. org/10.1590/S0104-11692008000200009

NUnNally, J.; BERnSteIN, I. Psychometric theory. 3rd ed. New York: McGraw-Hill, 1994. 752 p.

OLIVER, R. L. A cognitive model of the antecedents and consequences of satisfaction decisions. Journal of Marketing Research, v. 17, p. 460-469, 1980. http:// dx.doi.org/10.2307/3150499

OLIVER, R. L. Cognitive, affective, and attribute bases of the satisfaction response. Journal of Consumer Research, v. 20, p. 418- 430, 1993. http://dx.doi. org/10.1086/209358

OLIVER, R. L. Whence Customer Loyalty? Journal of Marketing, v. 63, n. 2, p. 33-44, 1999. http://dx.doi. org/10.2307/1252099

OLSEN, S. Repurchase loyalty: the role of involvement and satisfaction. Psychology \& Marketing, v. 24, n. 4, p. 315-341, 2007. http://dx.doi.org/10.1002/mar.20163

PARASURAMAN, A.; ZEITHAML, V. A.; BERRY, L. L. A Conceptual Model of Service Quality and Its Implications for Future Research. Journal of Marketing, v. 49, n. 4, p. 41-50, 1985. http://dx.doi.org/10.2307/1251430

PARASURAMAN, A.; ZEITHAML, V. A.; BERRY, L. L. SERVQUAL: a multiple-item scale for measuring consumer perceptions of quality. Journal of Retailing, v. 63 , n. 1 , p. $12-37,1988$.

PARASURAMAN, A.; GREWAL, D.; KRISHNAN, R. Marketing research. 2nd ed. South Western College Pub, 2006.

PEREIRA, D. R. M.; PINTO, M. R. A Teoria da Resposta ao Item: possíveis contribuições aos estudos em marketing. Gestão \& Produção, v. 18, n. 4, 2011. http://dx.doi. org/10.1590/S0104-530X2011000400010

POWERS, D.; XIE, Y. Statistical methods for categorical data analysis. London: Academic Press, 2000.

RAYKOV, T.; MARCOULIDES, G. A. A First Course in Structural Equation Modeling. Mahwah: Lawrence Erlbaum, 2000.

REICHHELD, F. F.; SASSER, W. E. Zerodefections: Quality comes to services. Harvard Business Review, v. 68, n. 5, p. 105-11, 1990.

RIBEIRO, J. L. D.; MACHADO, C.O.; TINOCO, M. A. C. Determinantes da satisfação e atributos da qualidade em serviços bancários. Gestão \& Produção, v. 17, n. 4, p. 775-790, 2010. http://dx.doi.org/10.1590/ S0104-530X2010000400011

RUYTER, D.; KO, L. M.; LEMMINK, J. Antecedents of commitment and trust in customer-supplier relationships in high technology markets. Industrial Marketing Management, v. 30, n. 2, p. 271-286, 2001. http:// dx.doi.org/10.1016/S0019-8501(99)00091-7

SALOMI, G. G. E.; MIGUEL, P. A. C; ABACKERLI, A. J. SERVQUAL x SERVPERF: comparação entre instrumentos para avaliação da qualidade de serviços internos. Gestão \& Produção, v. 12, n. 2, ago. 2005.

SEIDERS, K. et al. Do satisfied customers buy more? Examining moderating influences in a retailing context. Journal of Marketing, v. 69, p. 26-43, Oct 2005. http:// dx.doi.org/10.1509/jmkg.2005.69.4.26

SELNES, F.; GONHAUG, K. Effects of reliability and benevolence in business marketing. Journal of Business Research, v. 49, n. 3, p. 259-271, 2000. http://dx.doi. org/10.1016/S0148-2963(99)00017-X

STANK, T. P.; GOLDSBY, T. J.; VICKERY, S. K. Effect of service supplier performance on satisfaction and loyalty of store managers in the fast food industry. Journal of Operations Management, v. 17, n. 4, p. 429-448, 1999.

STANK, T. P. et al. Logistics service performance: estimating its influence on market share. Journal of Business Logistics, v. 24, n. 1, p. 27-56, 2003. http://dx.doi. org/10.1016/S0272-6963(98)00052-7

STERLING, J. U.; LAMBERT, D. M. Establishing customer service strategies within the marketing mix. Journal of Business Logistics, v. 8, n. 1, p.1-30, 1987.

TONTINI, G.; ZANCHETT, R. Atributos de satisfação e lealdade em serviços logísticos. Gestão \& Produção, v. 17, n. 4, p. 801-816, 2010. http://dx.doi.org/10.1590/ S0104-530X2010000400013

TOO, L. H.; SOUCHON, A. L.; THIRKELL, P. C. Relationship marketing and customer loyalty in a retail setting: a dyadic exploration. Journal of Marketing Management, v. 17, n. 3-4, p. 287-320, 2001.

VARGAS, V. C. C. et al. Avaliação dos intangíveis: uma aplicação em capital humano. Gestão \& Produção, v. 15, n. 3, dez. 2008. http://dx.doi.org/10.1590/ S0104-530X2008000300015

VIEIRA, A. L. ABC do LISREL Interativo: Um Exemplo Prático de Modelação em Equações Estruturais. Lisboa: Silabo 2009.

WEST, S. G.; FINCH, J. F.; CURRAN, P. J. Structural equation models with nonnormal variables: problems and remedies. In: HOYLE, R. H. (Ed.). Structural Equation Modeling: concepts, issues and applications. London: Sage Publications Inc., 1995. cap. 4, p. 56-75.

WETZELS, M.; RUYTER, K.; BIRGELEN, M. Marketing service relationships: the role of commitment. Journal of Business and Industrial Marketing, v. 13, n. 4-5, p. 406-423, 1998. http://dx.doi. org/10.1108/08858629810226708 
Anexo 1. Definição operacional das variáveis.

\begin{tabular}{|c|}
\hline Medidas \\
\hline $\begin{array}{l}\text { Desempenho Operacional } \\
\text { 1. Quão eficiente e eficaz é o processo de colocar pedidos para este fornecedor? } \\
\text { 2. Quão hábil é o fornecedor para corrigir as discrepâncias dos pedidos após o recebimento dos produtos? } \\
\text { 3. Avalie o tempo entre a colocação do pedido e a data de chegada do produto. } \\
\text { 4. Avalie o tempo entre a colocação do pedido e a data de chegada do produto para pedidos especiais. } \\
\text { 5. Avalie a consistência do tempo entre a colocação do pedido e a data de chegada do produto. } \\
\text { 6. Avalie quanto o produto é entregue na, ou antes, da data solicitada. } \\
\text { 7. Avalie a disponibilidade e habilidade de se obter as quantidades solicitadas no pedido. } \\
\text { 8. Avalie quanto os embarques atendem a seus pedidos depois de sua efetiva chegada: quantidades e produtos } \\
\text { corretos, sem substituições. } \\
\text { 9. Qual a qualidade dos produtos entregues, ou seja, embarques livres de avarias. }\end{array}$ \\
\hline $\begin{array}{l}\text { Desempenho Relacional } \\
\text { 1. Eles tentam entender sua situação individual. } \\
\text { 2. Eles são responsivos diante de qualquer problema que apareça. } \\
\text { 3. Eles trabalham em conjunto com você para tornar o atendimento a pedidos mais eficiente. } \\
\text { 4. Eles fazem recomendações para melhoria contínua de forma consistente. } \\
\text { 5. Eles conhecem bem suas necessidades. } \\
\text { 6. Eles possuem conhecimento do seu negócio. } \\
\text { 7. Eles são proativos e informam se o pedido sofrerá algum atraso. }\end{array}$ \\
\hline $\begin{array}{l}\text { Satisfação } \\
\text { 1. Estou contente realizando negócios com eles. } \\
\text { 2. Estou muito satisfeito com os serviços prestados por eles quando comparados com a nossa expectativa. } \\
\text { 3. No geral eu estou muito satisfeito com os serviços prestados por eles. } \\
\text { 4. Eles fornecem completamente todos os serviços que eu quero deles. } \\
\text { 5. Os serviços prestados por eles estão muito próximos da perfeição. } \\
\text { 6. Eles fornecem serviços quase inaceitáveis. } \\
\text { 7. Destaca-se de outros fornecedores pelo nível superior de seus serviços. }\end{array}$ \\
\hline
\end{tabular}

\section{Lealdade Atitudinal}

1. Eu tenho desenvolvido um relacionamento de negócios mais próximo com este fornecedor do que com outros fornecedores.

2. Eu realmente gosto de realizar negócios com este fornecedor, até mais do que com outros fornecedores.

3. Eu estou mais disposto a adquirir mais produtos deles do que de outros fornecedores.

4. De todas as empresas desse mercado com as quais minha empresa faz negócios, fazer negócios com este fornecedor é muito mais importante.

5. Eu quero me manter como cliente deste fornecedor muito mais do que com outros fornecedores porque nós realmente apreciamos o relacionamento que temos com eles.

6. Eu estou muito mais comprometido com eles do que com outros fornecedores.

\begin{tabular}{l} 
Mealdade Comportamental \\
\hline 1. Eu consistentemente compro mais regularmente deles do que de outros fornecedores. \\
2. Eu estou mais propenso a continuar realizando negócios com eles do que com os demais fornecedores do setor. \\
3. Eu tenho comprado mais deles durante os últimos anos do que dos demais fornecedores. \\
4. Eu o considero minha primeira opção. \\
5. Ele tem sido minha primeira opção durante os últimos anos. \\
6. Eu espero que ele seja a primeira opção no futuro.
\end{tabular}

